\title{
Performance Analysis of Visible Light Communications-Based Hotspots in Indoor and Outdoor Environments
}

\author{
Helal Chowdhury · Ari Pouttu • Marcos \\ Katz
}

Received: date / Accepted: date

\begin{abstract}
In this work, we study the performance of optical-based wireless (OW) hotspots in case of indoor and outdoor environments. The considered hotspots, also known as visible light communication (VLC) hotspots where visible light spectrum is used for data transmission. In prior to deploy of any wireless networks, a number of scenarios are targeted to evaluate the performance in terms of the capacity, reliability or any other selected performance metric. However, to evaluate the performance of optical-based wireless networks, along with the impact of optical predominated obeying line-of-sight (LOS) propagation other parameters such as in point-to-point link alignment between transmitter and receiver, field of view (FOV) of the receiver, semiangle of transmitter, background noise such as ambient and thermal noise, velocity of the user are also need to be considered. In this paper, we use the throughput-distance relationship model which has been derived analytically by taking into account above mentioned parameters for evaluating the performance of VLC hotspot networks in indoor and outdoor environments at both, day and night conditions. Simulations are also performed to evaluate the performance of such hotspot network in the context of data download on move scenario. Simulation results reveal that there is a large impact of background noise on the performance of VLC hotspots. As expected, in both indoor and outdoor environments VLC hotspot performs better at night than at day time. Performance of VLC hotspot networks is also quantified in terms of received file size at different bit error rate requirements and velocities of mobile user. For example, in a typical indoor environment at night time a mobile user with
\end{abstract}

H. Chowdhury

Centre for Wireless Communications, University of Oulu, Finland, Tel.: +358-40-5297862

E-mail: helal.chowdhury@ee.oulu.fi

A. Pouttu

Centre for Wireless Communications University of Oulu, Finland E-mail: apo@ee.oulu.fi

M. Katz

Centre for Wireless Communications University of Oulu, Finland E-mail: mkatz@ee.oulu.fi 
a BER requirement of $10^{-3}$ at velocity $1 \mathrm{~m} / \mathrm{sec}$ can download 4 megabyte file from a particular designed of VLC hotspot.

Keywords Visible Light Communications · Optical Hotspots · Optical Wireless Communications - Throughput-distance Relationship Model . Performance Analysis

\section{Introduction}

Solid-state-based light emitting diode (LED) lighting sources are rapidly becoming the mainstream for illumination solutions. It is predicted that for illumination all lighting will be based on light-emitting diode in the near future because of its energy efficiency and reliability compared to existing lighting solutions [1]. It is expected that visible light communication (VLC) will also receive a huge momentum in the upcoming years [1]. VLC is a particular case of optical wireless communications where the visible part of the optical spectrum is used for communications purposes. The visible light spectrum extends from (380-780) $\mathrm{nm}$ in wavelength. This very broad and unlicensed optical spectrum of VLC can also be thought as an alternative solution for spectrum scarcity where it can provide access to several hundred terahertz of unlicensed spectrum $[2,14]$. In such case, the traffic load of wireless fidelity (WiFi) and cellular femto cells can be offloaded to the optical bands by exploiting optical air interfaces. The other key merits of optical wireless systems include the relatively low transceiver complexity and low energy-per-bit required for data transmission in comparison to radio frequency ( $\mathrm{RF}$ ) based systems.

In VLC, white LEDs are used as transmitters; the air is the optical transmission medium, and the appropriate photodiodes or image sensors are used as signal receiving components. As a result, illumination and communications can be achieved with a single platform. There are already several standards associated with visible light communications [15,16]. In 2011, VLC was standardized and published as the IEEE 802.15.7 standard. This standard promises to be a very attractive candidate as a future high data rate and power-efficient technology [12]. In such case, future solid-state lighting infrastructure can be used as a high data-rate VLC-based hotspots. VLC-based hotspots is also known as visible hotspots because of its coverage area is illuminated [1,4]. Optical hotspots can be built in indoor as well as outdoor environments. Visible hotspots are expected to reach office spaces, homes, street and traffic lights, shops and others.

However, deployment of mobile communication via VLC is challenging due to, among others, occlusion which can occur due to severe misalignments and presence of physical obstruction between sources and detectors in indoor as well as outdoor environments [1]. Visible light waves predominantly obey lineof-sight (LOS) propagation. Hence, VLC networks can be considered as obstructed wireless networks or so called line-of-sight networks [13]. Therefore, there are significant challenges for circumventing the LOS nature of VLC link. Moreover, VLC performance also depends on some external factors, such as 
ambient noise, the field of view (FOV) of the concentrator, the semiconductor material used in the transmitter and receiver and so on. This advocates the need to study the VLC link characteristics for development, validation, and analysis of quantitative models which will ultimately help to design such obstructed or line-of-sight networks before being deployed in practice.

In this work we provide a methodology to evaluate the performance of visible hotspot networks in the context of data downloading on the move by using throughput-distance relationship models [20]. Performance evaluation of wireless local area network (WLAN) and relay-based networks using throughput-distance models are done and reported in $[9,20]$. In contrast to the related works, this work mainly focuses on obstructed networks, that is, VLC networks where throughput depends on many optical constraints. As mentioned, the affecting factors include alignment between the transmitter and receiver in point-to-point link, semi-angle of transmitter, receiver FOV, transmit power of the LED, photo detector area, state of the channel, noise, interference produced by many artificial lights and so on. The effect of all these parameters to the throughput can be approximated and modeled as a throughput-distance relationship model [20]. The main contributions of this work is to derive the throughput-distance relationship model for VLC hotspot networks. The throughput-distance relationship is then used to evaluate the performance of VLC networks in indoor and outdoor environments. The obtained results and proposed methodologies provide a fundamental information to design and deployment related issues of VLC hotspots.

The remaining of this paper is structured as follows. In Section 2 optical wireless LOS link characteristics is identified. In Subsection 2.2 noise characteristics of optical wireless system in indoor and outdoor environment are discussed. Mathematical frame work for data downloading scenario is presented in Section 3. Throughput-distance relationship development is presented in Section 4. Random mobility of mobile user is also discussed in Subsection 4.2. Simulation results and discussion are reported in Section 5. Finally conclusions are drawn in Section 6.

\section{Optical wireless LOS link characteristics}

In optical wireless communications (OWC) radiant intensity $R_{i}$ for LOS propagation model can be expressed as [19]:

$$
R_{i}=P_{t} R_{0}(\phi)
$$

where $P_{t}$ is transmitted power and $R_{0}(\phi)$ is the Lambartian pattern. Lambartian pattern at incidence angle $\phi$ can be expressed as $[19,21]$

$$
R_{0}(\phi)=\frac{m+1}{2 \pi} \cos ^{m}(\phi)
$$


where $m$ is the order of Lambertian radiation pattern, $m$ is related to the LED semiangle at half-power $\Psi_{1 / 2}$ given by $[19,21]$

$$
m=\frac{\ln 2}{\ln \left(\cos \left(\Psi_{1 / 2}\right)\right)} .
$$

It should be noted that $R_{0}(\phi)$ is a function of two angles: angle of incidence $\phi$ and half power semi angle $\psi_{1 / 2}$. Without considering reflections, direct current (DC) gain $\mathrm{H}(0)$ in LOS channel can be expressed as [21]

$$
H(0)=\left\{\begin{array}{l}
\frac{(m+1) A_{d}}{2 \pi D^{2}} \cos ^{m}(\phi) T_{s}(\psi) g(\psi) \cos (\psi), 0 \leq \psi \leq \Psi_{c} \\
0, \quad \psi>\Psi_{c},
\end{array}\right.
$$

where $D$ is the distance between LED and photo detector (PD), $A_{d}$ is the detector area, $T_{s}(\psi)$ is the signal transmission of the filter, $g(\psi)$ is the concentrator gain and $\Psi_{c}$ is the concentrator FOV can be represented as semiangle.

Concentrator gain $g(\psi)$ of an ideal non-imaging concentrator with refractive index $k$ can be expressed as [21]

$$
g(\psi)=\left\{\begin{array}{l}
\frac{k^{2}}{\sin ^{2}\left(\Psi_{c}\right)} 0 \leq \psi \leq \Psi_{c} \\
0, \quad \psi>\Psi_{c}
\end{array}\right.
$$

In case of without receiver front end design the effective collection area of the PD can be represented as [19]

$$
A_{f}=\left\{\begin{array}{l}
A_{d} \cos (\phi),|\phi|<\mathrm{FOV} \\
0, \quad|\phi| \geq \mathrm{FOV}
\end{array}\right.
$$

2.1 Noise characteristics of optical wireless system in indoor and outdoor environments

The performance of wireless optical system is limited by various types of noise and interferences $[17,18]$. However, the two dominant sources of noise which affects the optical receiver performance are shot noise and thermal noise [21]. Shot noise, also referred to as ambient noise, is usually produced by natural and artificial light sources such as sunlight, incandescent lamps and fluorescent lamps. Thermal noise is generated by resistive element of the receiver circuit, it is also known as circuit noise [17]. On the other hand, interference is produced by many other different types of light sources such as incandescent lamps with tungsten filaments, halogen and mercury lamps, fluorescent lamps with different emitting color $[17,18]$. In this work, we mainly consider shot noise where background irradiance produced by natural and artificial light sources. The effect of this background irradiance is modeled by DC currents. The composition of all these currents are usually refereed to as background current $I_{b}$ which can be expressed as [21]

$$
I_{b}=I_{\text {natural }}+I_{\text {artificial }},
$$


Table 1 Background Noise $I_{b}$

\begin{tabular}{ccc}
\hline \hline Source & $\begin{array}{c}\text { Without optical filter } \\
(\mu A)\end{array}$ & $\begin{array}{c}\text { With optical filter } \\
(\mu A)\end{array}$ \\
\hline Direct sun light & 5100 & 1000 \\
Indirect sun light & 740 & 190 \\
Incandescent light & 84 & 56 \\
Fluorescent light & 40 & 2 \\
& & \\
\hline
\end{tabular}

where $I_{\text {natural }}$ and $I_{\text {artificial }}$ are the DC currents produced by natural and artificial light sources respectively. The shot noise is independent of the signal and can be modeled as a white Gaussian with one-sided power spectral density (PSD) $N_{0}$ given by $[21]$

$$
N_{0}=2 q I_{b},
$$

where $q$ is the electron charge. Measurement based background noise provided by natural and artificial light source in case of with and without optical filter in the receiver side is given in Table $1[17,18]$.

In general, signal to noise ratio (SNR) is used to express the quality of performance in communication systems. In such case, the performance of wireless optical link at the bit rate $R_{b}$ can be related to electrical SNR as [19]

$$
\mathrm{SNR}=\frac{R_{r}^{2} H^{2}(0) P_{t}^{2}}{R_{b} N_{0}},
$$

where $R_{r}$ is the responsivity of the photo detector.

We assume that the transmitter transmits the signal using on-off keying (OOK) modulation technique. BER of OOK can be expressed as

$$
\mathrm{BER}=Q(\sqrt{\mathrm{SNR}}) .
$$

The function $Q(x)$ is defined as

$$
Q(x)=\frac{1}{\sqrt{2 \pi}} \int_{x}^{\infty} e^{-}\left(\frac{y^{2}}{2}\right) d y .
$$

\section{Mathematical framework for a data downloading scenario}

The footprint of the coverage of LED source is shown in Figure 1. We assume that the access point (AP) is at coordinates $(0,0)$ and that the user enters the coverage at point $A^{\prime}$, with coordinates $\left(x_{a}, y_{a}\right)$ known as entering point and exits the coverage at point $B^{\prime}$, with coordinates $\left(x_{b}, y_{b}\right)$ known as exit point, as shown in Figure 1. For inclined path, the entrance angle is denoted with $\alpha_{1}$, and the exit angle is denoted with $\alpha_{2}$. The angle between entrance point and exit point is called traveling angle which is denoted as $\theta^{\prime}$. 


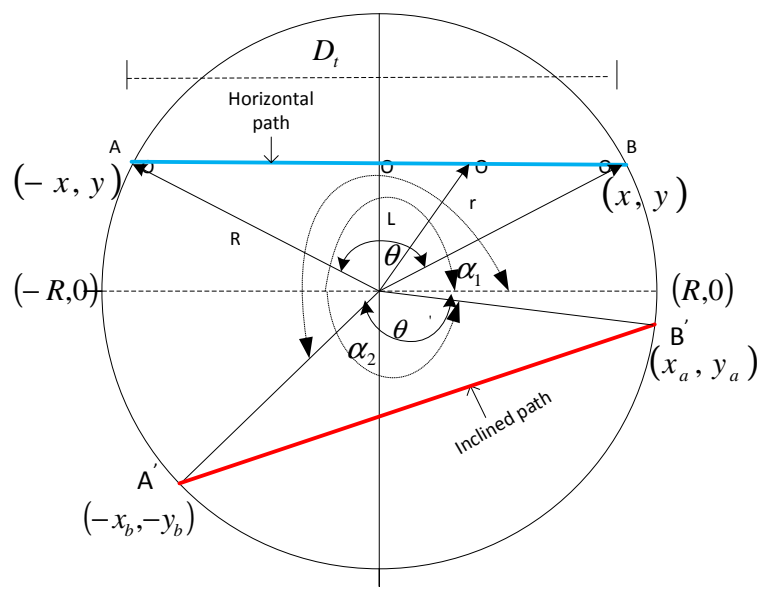

Fig. 1 Transformation of inclined path to horizontal path.

Assuming constant velocity, the path that the user travels can be simply expressed with

$$
(x(t), y(t))=\left(\left(x_{b}-x_{a}\right) t+x_{a},\left(y_{b}-y_{a}\right) t+y_{a}\right),
$$

where the parameter $t$ varies from zero (corresponding to the entrance point) to one (the exit point). Let us characterize the throughput as a function of the distance, i.e., the throughput at distance $r$ is $S(r)$. The instantaneous distance from the center of coverage to the mobile user can be calculated as

$$
r=\sqrt{\left[\left(x_{b}-x_{a}\right) t+x_{a}\right]^{2}+\left[\left(y_{b}-y_{a}\right) t+y_{a}\right]^{2}} d t .
$$

Now, we can find the average throughput of the user (while it passes through the coverage) with

$$
S_{a v}=\int_{t=0}^{1} S\left(\sqrt{\left[\left(x_{b}-x_{a}\right) t+x_{a}\right]^{2}+\left[\left(y_{b}-y_{a}\right) t+y_{a}\right]^{2}}\right) d t,
$$

where $S(\sqrt{\cdot})$ represents throughput-distance relationship function. This function can be either measurement- or analytical-based. In this work, we provide an analytical based throughput-distance model. Throughput-distance relationship model is discussed in Section 3. However the calculation of (14) for arbitrary entrance and exit points is cumbersome. Hence, in order to simplify the calculation of the average throughput we rotate the inclined path to an equivalent horizontal path as shown in Figure 1. In this case, entrance and exit coordinates points of inclined and horizontal path can be related to $x_{a}=x$, $x_{b}=-x, y_{a}=y y_{b}=y$. Moreover, the entrance and exit angle of inclined path can be transformed as $\alpha_{1}=\alpha$ and $\alpha_{2}=\alpha$. After transformation the average throughput over inclined path $\left(A^{\prime} B^{\prime}\right)$ will be the same as the calculation of the average throughput over the horizontal path $(A B)$. Depending on the 
difference between the entrance and exit angle of inclined path, after rotation traveling angle $\theta$ of the horizontal path can be expressed as [20]

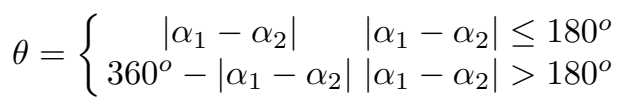

It should be noticed that the maximum range of traveling angle can be limited to the range of $\left(1^{0}-180^{\circ}\right)$ instead of $\left(1^{0}-360^{\circ}\right)$ for calculation of average throughput over the simplified horizontal path. The calculation of average throughput over the simplified horizontal path is given below:

After rotation, we get new entrance point $A(-x, y)$ and exit point $B(x, y)$, as shown in Figure 1. Hence for simplified horizontal path (13) can be written as

$$
r=\sqrt{[x(2 t-1)]^{2}+y^{2}} d t .
$$

In Figure 1, it is also shown that the minimum distance between mobile user and the AP is $L$, which for a particular traveling angle $\theta$ is $L=R \cos (\theta / 2)$, where $R$ is the coverage radius. The traveling distance $D_{t}=2 R \sin (\theta / 2)$. However, it should be noted that the traveling distance is equal before and after rotation (e.g $D=A^{\prime} B^{\prime}=A B$ ) that is the traveling angle of horizontal path $\theta$ is not affected by the rotation, that is $\left(\theta=\theta^{\prime}\right)$. Finally average throughput over the simplified horizontal path can be expressed as

$$
S_{a v}(r)=\int_{t=0}^{1} S\left(\sqrt{[x(2 t-1)]^{2}+y^{2}}\right) d t,
$$

where $x=\frac{D_{t}}{2}=R \sin (\theta / 2)$ and $y=L$. It is easily seen that (17) leads to much simplified integration in comparrson to (14). By taking velocity $v$ into account, the dwelling time of the mobile user in VLC hotspot can be found with $t_{d w e l l}=D_{t} / v=2 x / v$. Now by simply multiplying the dwell time with the average throughput, size of the transferred information or file size can be written as

$$
I_{t}(\theta)=S_{a v} \cdot t_{d w e l l} .
$$

Using (17) transferred information or file size can be written as follows:

$$
I_{t}(\theta)=\frac{2 x}{v} \int_{t=0}^{1} S\left(\sqrt{[x(2 t-1)]^{2}+y^{2}}\right) d t .
$$

\section{Throughput-distance model}

Signal coverage and data rate are the two important design issues in any wireless communication system. Determination of signal coverage and data rate is influenced by a variety of factors, most prominently the frequency of operation and the terrain [5]. In this case, path-loss model is used to calculate the 
signal coverage for any environment which relates the loss of signal strength to distance between transmitter and receiver. Throughput also is affected by the channel environment such as the distance between the transmitter and receiver. In point -to-point VLC link, alignment between transmitter and receiver, field of view (FOV) of the receiver, semi-angle of transmitter, background noise such as ambient noise. It may also influenced by the choice of design parameters, for example, modulation and coding, constellation size, power level, multiple access scheme, and many others. The effect of all these parameters to the throughput may be described as throughput-distance model. Before deriving relationship between throughput and distance. Let's represent (4) as

$$
H(0)=\frac{C_{c}}{D^{2}},
$$

where $C_{c}$

$$
C_{c}=\frac{(m+1) A_{d}}{2 \pi} \cos ^{m}(\phi) T_{s}(\psi) g(\psi) \cos (\psi) .
$$

In this work we have considered for two different environments (indoor, outdoor). Furthermore, each environment is conditioned into two classes: day time and night time. It should be noted that the effect of background noise will vary depending on the environment and its particular class. For specific value of BER and $N_{0}$ and using (9),(10), and (20) the relationship of data rate and distance $D$ can be expressed as

$$
R_{b}=\frac{R_{r}^{2} C_{c}^{2} P_{t}^{2}}{\left(Q^{-1}(\mathrm{BER})\right)^{2} N_{0} D^{4}} .
$$

Moreover, relationship between $R_{b}$ and distance $D$ can be represented as

$$
R_{b}=K \cdot D^{-4}
$$

where $\mathrm{K} \in\left\{\mathrm{K}_{\mathrm{in}}, \mathrm{K}_{\mathrm{id}}, \mathrm{K}_{\mathrm{on}}, \mathrm{K}_{\mathrm{od}}\right\}$ is a scaling factor, $\mathrm{K}_{\mathrm{in}}, K_{\mathrm{id}}, K_{\mathrm{on}}, K_{\mathrm{od}}$ represent the scaling factors of indoor night and indoor day time, outdoor night and outdoor day respectively, and is given as

$$
\mathrm{K}=\frac{R_{r}^{2} C_{c}^{2} P_{t}^{2}}{\left(Q^{-1}(\mathrm{BER})\right)^{2} N_{0}} .
$$

It should be noted that the transmit power and all other relevant parameters in (24) are kept constant both for indoor and outdoor environment except $N_{0}$ and BER. Moreover, we assume that the receiver has an optical filter, in such case, the effect of background noise with filter will be modeled according to Table 1 . The value of a scaling factor $\mathrm{K}$ for indoor and outdoor environments for different BER requirements is given in Table 2. 
Table 2 The Value of Scaling Factors for Indoor and Outdoor Environments

\begin{tabular}{llll}
$\mathrm{K}$ & \multicolumn{3}{c}{ BER } \\
& $10^{-} 3$ & $10^{-} 5$ & $10^{-} 6$ \\
$\mathrm{~K}_{\text {od }}$ & $0.47448 \times 10^{6}$ & $0.24911 \times 10^{6}$ & $0.20053 \times 10^{6}$ \\
$\mathrm{~K}_{\text {on }}$ & $8.6552 \times 10^{6}$ & $4.544 \times 10^{6}$ & $2.0053 \times 10^{6}$ \\
$\mathrm{~K}_{\text {id }}$ & $8.6552 \times 10^{6}$ & $4.544 \times 10^{6}$ & $2.0053 \times 10^{6}$ \\
$\mathrm{~K}_{\text {in }}$ & $0.8555 \times 10^{6}$ & $1.0627 \times 10^{6}$ & $2.0242 \times 10^{6}$
\end{tabular}

4.1 Average throughput and file size using piecewise throughput-distance model

In piecewise throughput-distance model, two co-centric circles are used to represent the two different types of throughput regions. For example, in Figure 2 the inner tier (tier 1) is used to represent the saturation level which is maximum throughput $S_{\max }$ and outer tier (tier 2) is used to represent the variable throughput region. In such case, our proposed piecewise throughput-distance model can be expressed as

$$
S(r)=\left\{\begin{array}{l}
S_{\max }, \quad 0<r<r_{i n} \\
K \cdot D^{-4}, \quad r_{i n}<r<R
\end{array}\right.
$$

where $r_{i n}=1 \mathrm{~m}$. Using (22), (25) and Table 2 throughput-distance relationship in indoor and outdoor for day and night conditioned is shown in Figure 3 and Figure 4.

When the mobile user passes through the "Travelling Distance 1" in outer tier as shown in Figure 2 then using (17) and the (25) the average throughput can be calculated as

$$
S_{a v}=\left(K \times \frac{1}{2}\right)\left(\frac{\arctan \left(\frac{x}{y}\right)\left(x^{2}+y^{2}\right)+x y}{x y^{3}\left(x^{2}+y^{2}\right)}\right)
$$

We can express (26) in terms of the traveling angle $(\theta)$ by using $x=R \sin (\theta / 2)$ and $y=R \cos (\theta / 2)$.

$$
S_{a v}=\left(K \times \frac{1}{2}\right)\left(\frac{\left(\frac{\theta}{2}\right)+\sin \left(\frac{\theta}{2}\right) \cos \left(\frac{\theta}{2}\right)}{R^{4} \sin \left(\frac{\theta}{2}\right) \cos ^{3}\left(\frac{\theta}{2}\right)}\right) .
$$

When the user passes through both inner tier and outer tier, for example through "Travelling Distance 2", the instantaneous distance $r$ to the outer tier segment can be expressed as

$$
r=\sqrt{\left((1-t) x+t \cdot x_{c}\right)^{2}+y^{2}}
$$




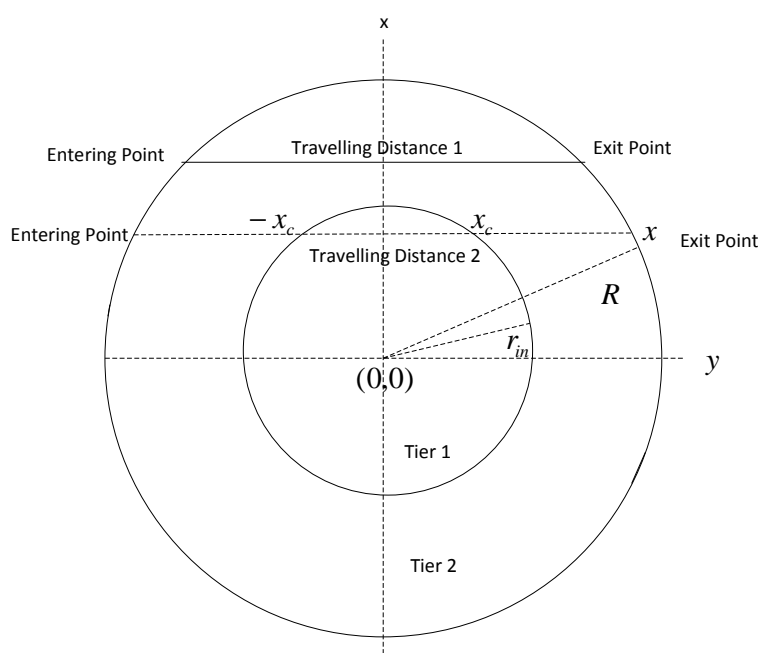

Fig. 2 Geometry of two-slope throughput distance model

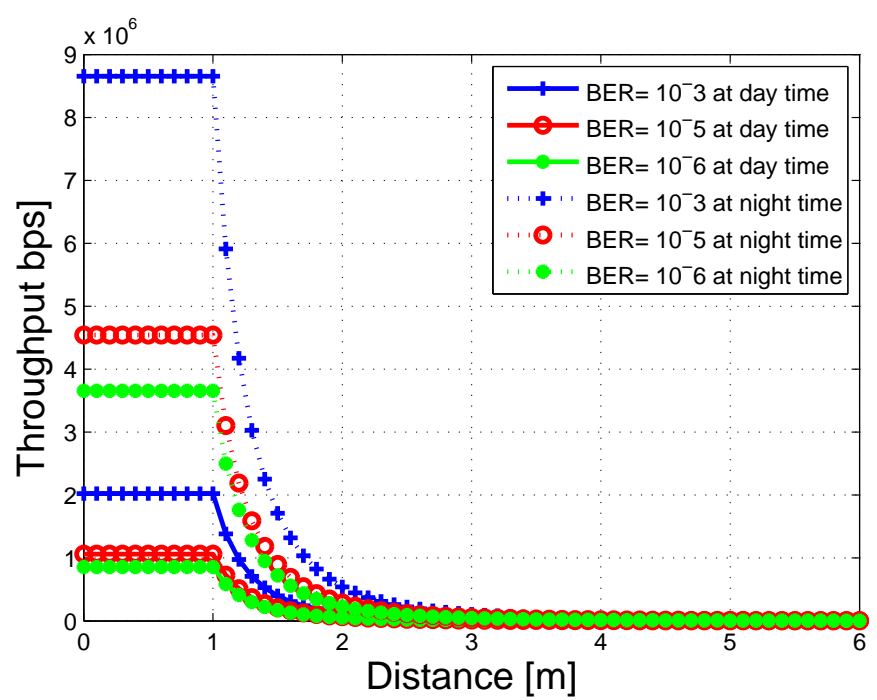

Fig. 3 Throughput vs. distance in an indoor environment.

In such case, average throughput in outer segment (in tier 2) can be calculated as

$$
\begin{aligned}
& S_{\mathrm{oc}}(r)=\frac{-1}{2\left(x_{c}-x\right)}\left(\left(x^{2}+y^{2}\right)\left(\arctan \left(\frac{x}{y}\right)-\arctan \left(\frac{x_{c}}{y}\right)\right)\right. \\
& \left.+y x-\frac{y x_{c}\left(x^{2}+y^{2}\right)}{x_{c}^{2}+y^{2}}\right) .
\end{aligned}
$$




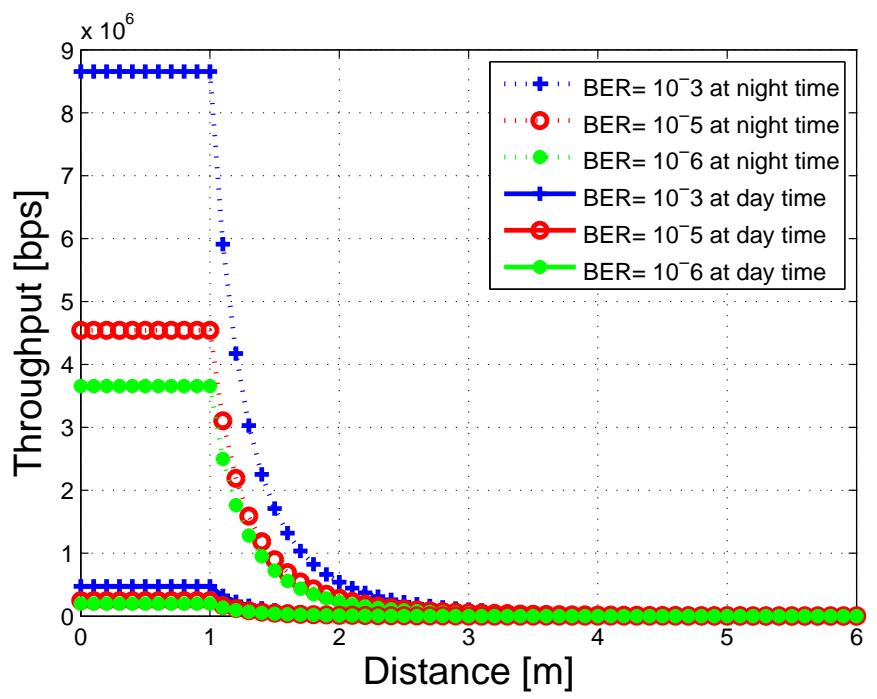

Fig. 4 Throughput vs. distance in an outdoor environment.

In terms of traveling angle, $\theta,(29)$ can be written as

$$
\begin{aligned}
& S_{\mathrm{OC}}(\theta)=\frac{-R^{2}}{2\left(x_{c}-R \sin \left(\frac{\theta}{2}\right)\right)}\left(\frac{\theta}{2}-\arctan \left(\frac{x}{R \cos \left(\frac{\theta}{2}\right)}\right)\right. \\
& \left.+\sin \left(\frac{\theta}{2}\right) \cos \left(\frac{\theta}{2}\right)-\frac{R \cos \left(\frac{\theta}{2}\right) x_{c}}{\left(x_{c}^{2}+R^{2} \cos ^{2}\left(\frac{\theta}{2}\right)\right.}\right) .
\end{aligned}
$$

Finally, when the user passes both through the outer and inner tier the average throughput can be calculated as:

$$
S_{\mathrm{av}}=\frac{S_{\mathrm{max}} x_{c}+S_{\mathrm{oc}}\left(x-x_{c}\right)}{x} .
$$

In such case average throughput in inner tier will be $S_{\max }$ and average throughput in outer tier will be $S_{\text {oc }}$

\subsection{Random mobility in VLC coverage}

In order to get an analytically tractable solution for calculating the average throughput and downloaded file size, we have considered constant velocity and unidirectional movement of the mobile user in Section 3. However, in practice, a mobility model should attempt to mimic the movement of a mobile user in reality. Most commonly used mobility model in the literature is the random waypoint model where user moves randomly, the destination, speed and direction are all chosen randomly in the course of its movement. It should be noted that in point-to-point communication, VLC networks can be considered as obstructed networks, so called line-of-sight networks [13]. Hence, when the mobile 
Table 3 Simulation Model Parameters

\begin{tabular}{|c|c|}
\hline Simulation Parameters & Value \\
\hline Transmit power & $20 \mathrm{~mW}$ \\
\hline Photodiode responsivity & 0.27 \\
\hline Semi-angle at half power & $60^{\circ}$ \\
\hline FOV at the receiver & $30^{\circ}$ \\
\hline Detector physical area of PD & $28 \mathrm{~mm}^{2}$ \\
\hline Coefficient of optical filter & 1.0 \\
\hline Mobile user velocity & {$[0.4-1] \mathrm{m} / \mathrm{s}$} \\
\hline BER & {$\left[10^{-} 3,10^{-} 5,10^{-} 6\right]$} \\
\hline Po & {$[0.05,0.5]$} \\
\hline
\end{tabular}

user changes its direction, the orientation of the mobile device may changes the alignment between transmitter and receiver. In this case, the LED divergence angle and FOV of the receiver will also changes. Due to these change the link characteristics between transmitter and receiver also changes. In many cases the mobile device may lose the connection. In this work, we have considered the probability of obstruction (Po) as a simulation parameter. $P_{o}$ is defined as the percentage of obstruction during the course of its movement.

In random mobility, a velocity vector $v=(v, \theta)$ is associated itself with two parameters, the first parameter is the magnitude of velocity $v$ of mobile user itself and second parameter $\theta$ is the direction of angle. The positions of the mobile user is defined by two dimensional coordinates (x,y). At every $\Delta t$ time step, both the velocity and the position of the mobile user are updated. Updated velocity and its direction can be expressed as [22]

$$
\begin{aligned}
& x(t+\triangle t)=x(t)+v(t) \cdot \cos (\theta(t)) . \\
& y(t+\triangle t)=y(t)+v(t) \cdot \sin (\theta(t)) ;
\end{aligned}
$$

where $v$ is assumed to be uniformly distributed random variable. Moreover, we have assumed that mobile nodes will move in five possible different directions. We also consider that each mobile has a higher probability in moving in the same direction as the previous move. The assigned values of probabilities to each direction are as follows:

$$
p_{0}=0.7, p_{1}=0.1, p_{2}=0.05 .
$$

so that $p_{0}+2 \cdot p_{2}+2 \cdot p_{2}=0.05$

\section{Simulation results and discussions}

In this Section we evaluate the performance of VLC communication system for indoor as well as for outdoor environments. Numerical results are analytically derived using the throughput-distance model to compare the performance of such network in day and night time. Monte-Carlo simulation results are carried out for 1000 iterations for constant mobility along with other system design 


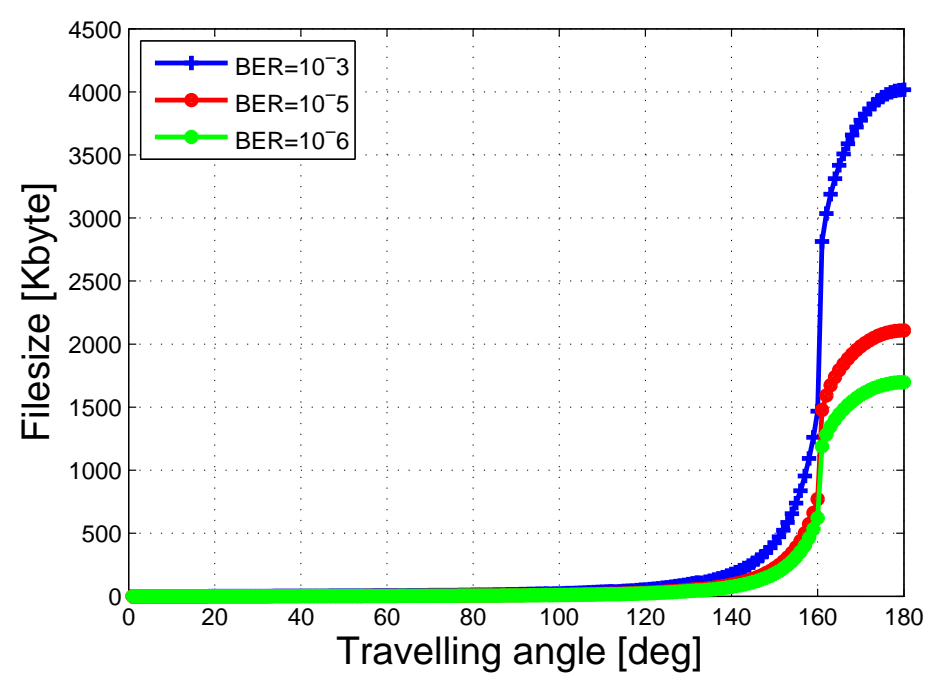

Fig. 5 File size vs. travelling angle in an indoor environment at night time.

parameters as given in Tables 1 and 2. A scenario of random velocity of user with given probability of obstruction is also considered for simulation as discussed in Subsection 4.2. The received file size by a mobile user serves as a performance metric.

Figure 5 shows the received file size of mobile user in VLC coverage for indoor environment at night time for different BER requirements. It is seen that at low required BER $\left(10^{-3}\right)$ the mobile user will have a larger file size than at higher BER $\left(10^{-5} \& 10^{-6}\right)$ requirements. At night time, there will be zero solar radiation. As a result, only artificial light sources such as fluorescent and tungsten light bulb will contribute in background noise. It is seen that file downloading performance at all BER requirements improves when the traveling angle is about $130^{\circ}$ which is basically the $2.5357 \mathrm{~m}$ from the center of VLC coverage. So, the coverage of the reasonable higher data rate region is from the center of VLC coverage to a distance $2.5357 \mathrm{~m}$. In another way, it can be said that as the traveling angle increases the size of the downloadable file also increases and this increment is noticeable when the mobile user passes in the range of traveling angles $\left(130^{\circ}-179^{\circ}\right)$. This happens because of longer residence time occurs at higher traveling angle of the mobile user in the VLC hotspot coverage. Mobile user can download around 4000 Kbyte at BER = $10^{-3}$ when the traveling angle is around $180^{\circ}$. At traveling angle $180^{\circ}$, the downloaded file size by mobile user will be around over 2000 Kbyte and over 1500 Kbyte for $\mathrm{BER}=10^{-5}$ and $\mathrm{BER}=10^{-6}$ respectively. With the observed average throughput a music file size of 900 Kbyte of stereo 8 bit AM radio sound quality can be downloaded at BER requirement $10^{-3}$ if the mobile user moves in the range of traveling angles $\left(170^{\circ}-180^{\circ}\right)$ at velocity $v=1 \mathrm{~m} / \mathrm{sec}$. 


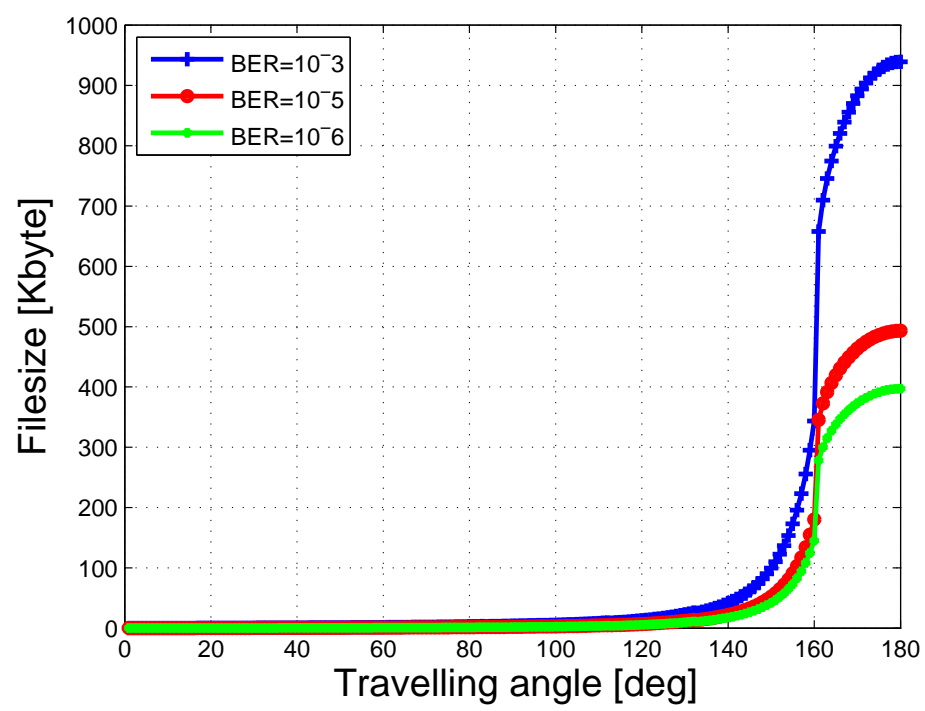

Fig. 6 File size vs. travelling angle in an indoor environment at day time.

Figure 6 shows the received file size in VLC coverage for an indoor environment at day time for three different BER requirements. It is clearly seen that the performance decreases drastically at all BER levels in comparison to the indoor environment performance at night time. This performance degradation occurs because of the impact of indirect solar radiation on the receiver at day time. It is seen from Table 1 that it is $190 \mu \mathrm{A}$ which is much higher natural source generated indirect noise background current in comparison to artificial source generated background current. The maximum downloadable file size is about 900,500 and 400 Kbyte at BER $\left(=10^{-} 3,=10^{-} 5,=10^{-6}\right)$ respectively. It is also seen that there is flat file size in lower traveling angle and higher file size in higher traveling angles. With the observed average throughput a music file size of 900 Kbyte of stereo 8 bit AM radio sound quality only can download at $\mathrm{BER}=10^{-3}$ if the mobile user moves with traveling angles between $\left(170^{\circ}-180^{\circ}\right)$ at velocity $v=1 \mathrm{~m} / \mathrm{sec}$. But at day time in indoor environment higher BER requirement applications cannot be downloaded more than 240 Kbyte at BER $10^{-5}$ and 170 Kbyte at BER $10^{-6}$.

Figure 7 shows the received file size in VLC coverage for an outdoor environment in night time for three different BER requirements at a velocity of 10 $\mathrm{m} / \mathrm{sec}$. In this case, the mobile user will download data while he is traveling by mobile vehicle. At this higher speed, the mobile user residence time will be considerably shorter, as a result the download file size will be very less in comparison to at night time in indoor environment. In such case, small file size applications such as downloading a city map can be provided to high speed mobile user. It is seen that a maximum of around 400, 210, and 170 Kbyte of data can be downloaded at the highest traveling angle. 


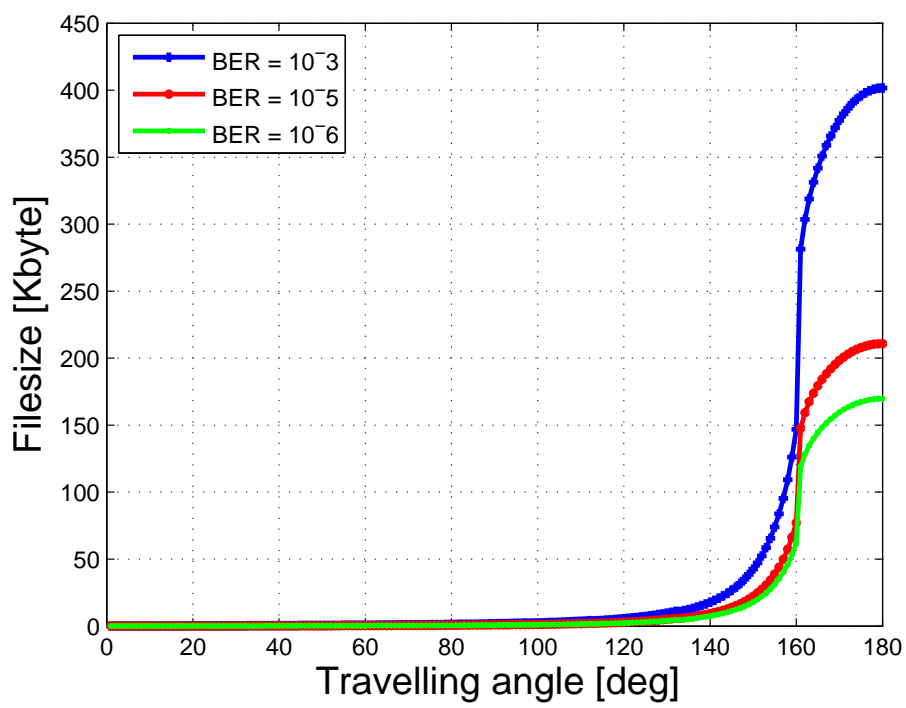

Fig. 7 File size vs. travelling angle in an outdoor environment at night time.

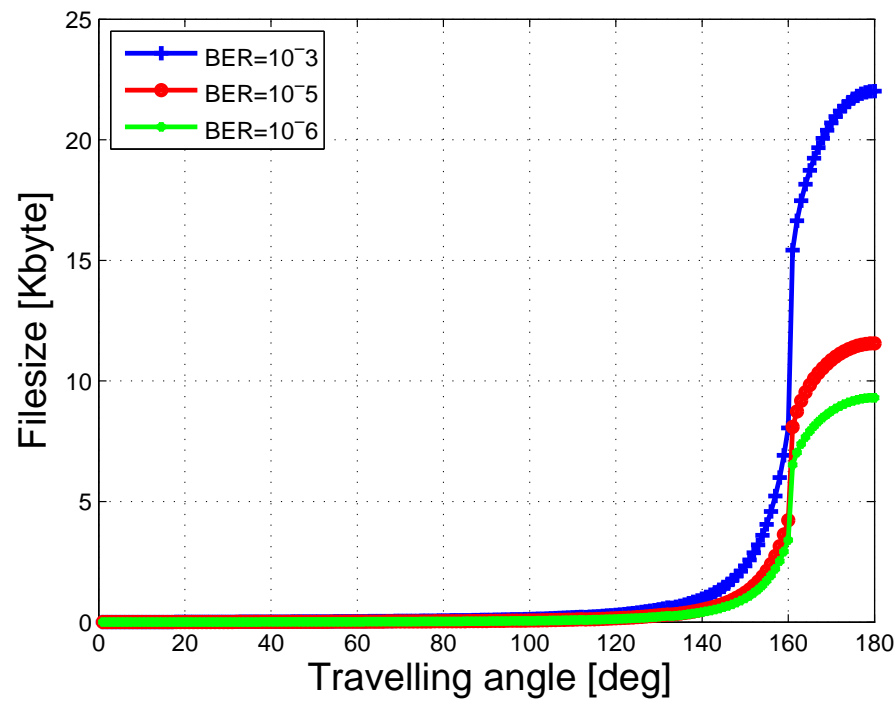

Fig. 8 File size vs. travelling angle in an outdoor environment at day time. 


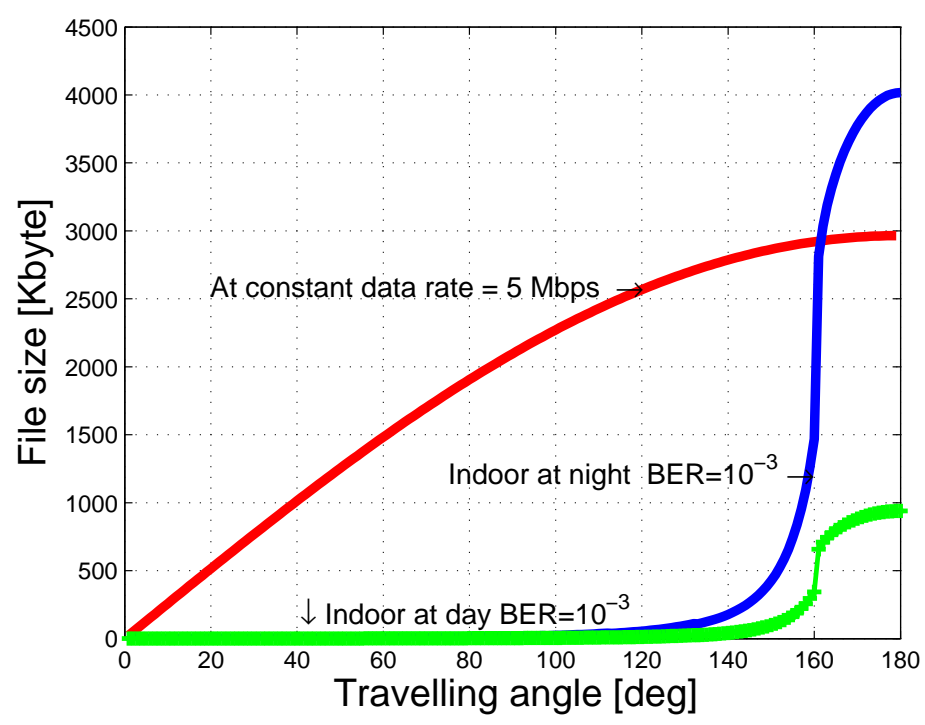

Fig. 9 Comparison between constant vs. variable data rate service.

Figure 8 shows the received file size in a single VLC hotspot coverage for an outdoor environment at day time for three different BER requirements at velocity of $10 \mathrm{~m} / \mathrm{sec}$. So, in case of a high speed user $(v=10 \mathrm{~m} / \mathrm{sec})$ the maximum file size that can be downloaded will be 23 Kbyte, 11 Kbyte and 9 Kbyte at BER requirement of $10^{-3}, 10^{-5}$, and $10^{-6}$ respectively. The reason for this degraded performance is the impact of direct solar radiation of sunlight and the higher speed of the mobile user. It is stated that in direct sunlight the background noise current is $1000 \mu \mathrm{A}$, this ambient noise has a huge impact on the outdoor environment at day time.

Figure 9 shows a comparison between constant data rate and variable data rate hotspots. As we have noticed from Figures (5-8) the mobile user is able to get file of considerable sizes when he passes in the range of $\left(130^{\circ}-180^{\circ}\right)$ travelling angle. On the other hand, at lower travelling angles the size of the files is negligible. However in case of VLC, LEDs may arrange in the LED panel in such way that it provides uniform data rate throughout the coverage (for example circular arrangement). Figure 9 shows a comparison of such constant data rate and variable data rate hotspot. It is clearly seen that constant data rate VLC hotspot outperforms variable data rate VLC hotspot in the lower range of travelling angles.

Figure 10 shows the cumulative distribution function (CDF) plots of file size for two different values of probability of obstruction $P_{o}=[0.05,0.5]$ and random velocity between $[0.4-1] \mathrm{m} / \mathrm{sec}$ for 10 mobility slots. For visualization and results analysis purposes we zoom the CDF plot. Simulation results have been obtained for night time in indoor environment scenarios. It is obvious that when the mobile user terminal is obstructed the performance of downloading 


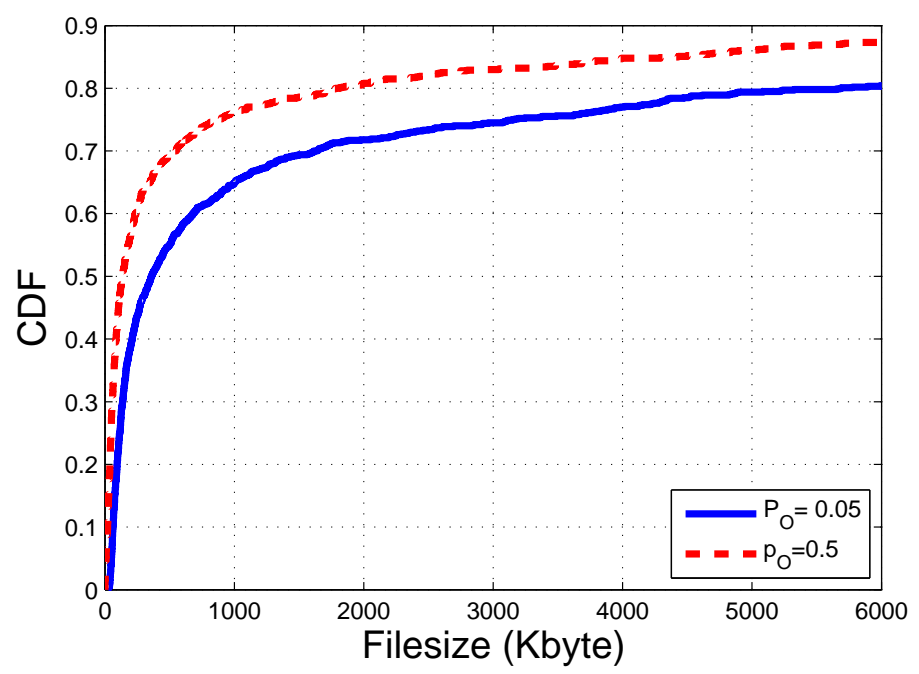

Fig. $10 \mathrm{CDF}$ vs. File size in random mobility with obstruction probability $P_{O}$.

file size also decreases. In such case there will be no data to be downloaded into the mobile user digital storage. As a result, the higher probability of obstruction the less data will be downloaded, a clearly expected result. It is also seen from Figure 10 that with $50 \%$ and probability of obstruction $(P o=0.05)$ there is chance to download data less than or equal to 358.6 Kbyte. On the other hand, with same probability and $P o=0.50$ there is chance to download data less than or equal to only 138.7 Kbyte.

\section{Conclusions}

In this work we studied the performance of optical wireless hotspots (a.k.a. VLC hotspots), where visible light spectrum is used as a medium for communication purposes in local access points. In contrast to radio-based wireless networks, VLC networks are more sensitive to environmental factors as well as other optical constraints such as alignment between transmitter and receiver, field of view (FOV) of the receiver, semi-angle of transmitter, state of the optical channel and different background noise at day and night time. To evaluate analytically the performance of such wireless communication networks, the impact of the above mentioned factors needs be considered and modeled. Simplified models are typically used to make the analysis tractable, such as the throughput-distance relationship model used in this research. In fact, this work exploited this relationship to evaluate performance of VLC hotspot networks in indoor and outdoor environments, both at day and night conditions. In this work we provide a methodology based on throughput-distance relationship models to evaluate the performance of visible hotspot networks in scenarios 
where users download data on the move. The derived average throughputdistance model is used to calculate the file size that can be downloaded while mobile users move through the VLC hotspots. File size was one of the key performance metric used for the analysis. Simulation results reveal that there is a large impact of background noise on the performance of a VLC hotspot. As expected, the VLC performance in both indoor and outdoor environments is better at night than at day time. Performance of VLC hotspot networks are also quantified in terms of received file size at different velocities of mobile users. Numerical examples show that in indoor environments a mobile user moving at $1 \mathrm{~m} / \mathrm{sec}$ can download at night time a four megabyte file at a BER requirement of $10^{-3}$. Finally, we argue that the presented framework can be used as a mathematical tool for evaluating the performance of sparsely coverage-based networks such as VLC hotspot and for deployment related issues in data downloading scenarios.

\section{References}

1. L. Hanzo, H. Haas, S. Imre, D. O'Brien, M. Rupp, and L. Gyongyosi, "Wireless myths, realities, and futures: From $3 \mathrm{G} / 4 \mathrm{G}$ to optical and quantum wireless," Proceedings of the IEEE, vol. 100, no. Special Centennial Issue, pp. 1853-1888, 2012.

2. D. O’Brien, M. Katz, P. Wang, K. Kalliojarvi, S. Arnon, M. Matsumoto, R. Green, and S. JIvkova, "Short-range optical wireless communications," pp. 1-22.

3. IEEE1, "IEEE standard for local and metropolitan area networks-part 15.7: Short-range wireless optical communication using visible light," IEEE Std 802.15.7-2011, pp. 1 -309, 62011.

4. S. Rajagopal, R. Roberts, and S.-K. Lim, "Ieee 802.15 .7 visible light communication: modulation schemes and dimming support," Communications Magazine, IEEE, vol. 50, no. 3, pp. $72-82$, Mar. 2012.

5. K. Pahlavan and A. H. Levesque, Wireless Information Networks, 2nd ed. John Wiley \& Sons, 2005.

6. H. Chowdhury, J.-P. Makela, and K. Pahlavan, "Traffic statistics on the coverage of wlan-based music shower," in Personal, Indoor and Mobile Radio Communications, 2006 IEEE 17th International Symposium on, Sept. 2006, pp. $1-5$.

7. A. Sivabalan and J. John, "Improved power distribution in diffuse indoor optical wireless systems employing multiple transmitter configurations," Optical and Quantum Electronics, vol. 38, pp. 711-725, 2006.

8. M. G. Almiron, O. Goussevskaia, A. A. Loureiro, and J. Rolim, "Connectivity in obstructed wireless networks: from geometry to percolation," in Proceedings of the fourteenth ACM international symposium on Mobile ad hoc networking and computing.

9. H. Elgala, R. Mesleh, and H. Haas, "Indoor optical wireless communication: potential and state-of-the-art," Communications Magazine, IEEE, vol. 49, no. 9, pp. 56-62, 2011.

10. CP-1221, Japanese Visible Light Communications System, 2nd ed. Japan Electronics Industry Technology Association, 2007.

11. CP-1222, Japanese Visible Light Communications System, 2nd ed. Japan Electronics Industry Technology Association, 2007.

12. A. J. C. Moreira, R. Valadas, and A. de Oliveira Duarte, "Characterisation and modelling of artificial light interference in optical wireless communication systems," in Personal, Indoor and Mobile Radio Communications, 1995. PIMRC'95. Wireless: Merging onto the Information Superhighway., Sixth IEEE International Symposium on, vol. 1, 1995, pp. 326-331 vol.1.

13. A. J. C. Moreira, R. T. Valadas, and A. M. de Oliveira Duarte, "Optical interference produced by artificial light," Wirel. Netw., vol. 3, no. 2, pp. 131-140, May 1997. 
14. J. Kahn and J. Barry, "Wireless infrared communications," Proceedings of the IEEE, vol. 85, no. 2, pp. 265-298, 1997.

15. H. Chowdhury, J. J. Lehtomäki, J.-P. Mäkelä, and S. Kota, "Data downloading on the sparse coverage-based wireless networks," J. Electrical and Computer Engineering, vol. 2010, 2010.

16. P. W. Ghassemlooy, Z. and A. H. Levesque, Optical Wireless Communications, 1st ed. CRC Press, 2013.

17. T. Camp, J. Boleng, and V. Davies, "A survey of mobility models for ad hoc network research," Wireless Communications Mobile Computing (WCMC): Special Issue on Mobile Ad Hoc Networking: Research, Trends and Applications, vol. 2, pp. 483-502, 2002. 